\title{
Utilization of E-cadherin by monocytes from tumour cells plays key roles in the progression of bone invasion by oral squamous cell carcinoma
}

\author{
JINGJING QUAN $^{1}$, QIAN DU ${ }^{1}$, YULUAN HOU ${ }^{1}$, ZHIYUAN WANG $^{2}$ and JINGYUAN ZHANG ${ }^{1}$ \\ ${ }^{1}$ Guanghua School of Stomatology, Hospital of Stomatology, Sun Yat-sen University and Guangdong Provincial \\ Key Laboratory of Stomatology, Guangzhou, Guangdong 510080; ${ }^{2}$ The Affiliated High School \\ of South China Normal University, Guangzhou, Guangdong 510630, P.R. China
}

Received December 14, 2016; Accepted May 17, 2017

DOI: $10.3892 /$ or.2017.5749

\begin{abstract}
E-cadherin (E-cad) is recently reported to be expressed in early stages of osteoclastogenesis, and blocking E-cad with neutralizing antibodies decreases osteoclast differentiation. Since our previous research demonstrates the loss of E-cad protein in the bone invasion by oral squamous cell carcinoma (OSCC), we hypothesize that E-cad may be utilized by monocytes to fuse and differentiate into osteoclasts. Two research models are used in the present study to explore our hypothesis. On one hand, we use OSCC cells of SCC25 to establish an animal model of bone invasion by OSCC, and investigate whether E-cad protein disappears in vivo; on the other hand, we use the indirect co-culture model of SCC25 and RAW 264.7 cells, with the treatment of transforming growth factor- $\beta 1$ (TGF- $\beta 1$ ), and observe whether the decreased E-cad protein is 'hijacked' in vitro. Results showed the animal model of OSCC with bone invasion was successfully established. Immunohistochemistry (IHC) found similar changes of E-cad protein, which was weakly stained by tumour cells. By using $5 \mathrm{ng} / \mathrm{ml}$ of TGF- $\beta 1$, we confirmed the artificial epithelial-mesenchymal transition (EMT) of SCC25 cells, with changes of EMT marker expression and cell morphology. Real-time PCR showed E-cad mRNA decreased in SCC25 while increased in RAW 264.7 of the indirect cell co-culture model, and immunofluoresence (IF) observed the evident switch of E-cad staining from SCC25 to RAW 264.7. With the supplement of receptor activator of NF- $\kappa$ B ligand (RANKL), tartrate-resistant acid phosphatase (TRAP) and F-actin staining confirmed the increased number of osteoclasts. Taken
\end{abstract}

Correspondence to: Dr Jingjing Quan, Guanghua School of Stomatology, Hospital of Stomatology, Sun Yat-sen University and Guangdong Provincial Key Laboratory of Stomatology, Guangzhou, Guangdong 510080, P.R. China

E-mail: quanjj3@mail.sysu.edu.cn

Key words: E-cadherin, osteoclasts, bone invasion, oral squamous cell carcinoma, epithelial-mesenchymal transition together, our study found the switch of E-cad protein in the progression of bone invasion by OSCC. The loss of E-cad in tumour cells may be utilized by monocytes to differentiate into osteoclasts, thus further explaining the underlying mechanisms of bone invasion by OSCC, which may supply clues for future molecular biotherapies.

\section{Introduction}

As one of the common complications, bone invasion is frequently diagnosed with the progression of bone invasion by OSCC $(1,2)$. Although local bone invasion and distant bone metastasis are closely related pathological processes, invasion of bone by OSCC has its own special characteristics (3). Initially, proteases help to degrade the extracellular matrix (ECM) of soft tissue and facilitate the entry of malignant keratinocytes. Secondly, osteoclasts are recruited by cytokines from tumour cells, and take the main role to resorb the bone. Lastly, osteoclasts mediate the bone resorption and liberate growth factors, promote the growth of neoplastic cells, thus driving a vicious cycle to accelerate the invasion (4).

Of the growth factors released, TGF- $\beta$ is embedded in the reservoirs of mineralized bone matrix and easily secreted during the degradation. TGF- $\beta$ is the TGF super family member, which normally controls tissue homeostasis by limiting cell proliferation (5). Three isoforms of TGF- $\beta$ have been identified in human: TGF- $\beta 1$, TGF- $\beta 2$, TGF- $\beta 3$. Since cancer cells usually have specific gene mutations or loss of TGF- $\beta$ signaling components; they can antagonize the inhibitory effects and selectively shut down its suppression arm (4). Additionally, TGF- $\beta$ has been proved to stimulate artificial EMT of epithelial cells, as well as malignant cells in vitro (6). Based on the well known report that induction of EMT by TGF generates cancer cells with higher stem cell properties, TGF has been utilized as a research tool to generate artificial EMT in a variety of studies (7). Previous work of our group has found that long-term treatment of recombinant human TGF- $\beta 1$ (rhTGF- $\beta 1$ ) triggers the EMT of OSCC cells in vitro (8). Typical changes include cell phenotype changing from slabstone to spindle shape, also with EMT marker expression changes of Snail, Slug, E-cad and N-cad. These 
EMT changes were further found to be associated with bone invasion of OSCC, and we suppose that TGF- $\beta 1$ may not only induce EMT to increase the invasive ability of OSCC cells, but also promote expression of osteoclastic factors and prolong osteoclast survival (9).

Recently, a report of osteoclast fusion machinery by Fiorino and Harrison found the protein of E-cad was expressed during early stages of osteoclastogenesis in both monocytes and primary macrophages (10). Blocking E-cad with neutralizing antibodies significantly diminished multinucleared osteoclast differentiation. E-cad-GFP overexpressing macrophages displayed rapid differentiation of mature osteoclasts. Since TGF- $\beta 1$ could induce artificial EMT of cancer cells with 'cadherin switch', and our previous research demonstrated the loss of E-cad protein in the progression of bone invasion by OSCC (9), we hypothesized that E-cad may be utilized by monocytes to fuse and differentiate into osteoclasts. Therefore, in the present study, we used two research models to explore our hypothesis. On one hand, we use OSCC cells of SCC25 to establish an animal model of bone invasion by OSCC, and investigated whether E-cad 'disappear' in vivo; on the other hand, we used the indirect cell co-culture model of SCC25 and RAW 264.7, with the treatment of TGF- $\beta 1$, to evaluate whether E-cad protein is 'hijacked' in vitro.

\section{Materials and methods}

Reagents. Dubecco's modified Eagle's medium (DMEM), $\alpha$-MEM, foetal bovine serum (FBS), trypsin-EDTA, antibiotics and phosphate buffered saline (PBS) were purchased from Thermo Fisher Scientific (Waltham, MA, USA). Primary antibody of mouse anti-human monoclonal E-cad (cat. no. 4A2) and N-cad (cat. no. 13A9) were obtained from Cell Signaling Technology, Inc. (Danvers, MA, USA), rabbit anti-human polyclonal Cytokeratin (CK, cat. no. BA2266-1) and Vimentin (Vim, cat. no. PB0378) from Boster (Wuhan, China), mouse anti-human monoclonal Snail1 (cat. no. sc-271977) was from Santa Cruz Biotechnology (Santa Cruz, CA, USA), and mouse anti-human monoclonal $\alpha$-tublin (cat. no. ab15246) was from Abcam (Cambridge, MA, USA). The secondary antibody with horseradish peroxidase (HRP)-conjugated (cat. nos. STAR137P and STAR121P) was supplied by Bio-Rad Laboratory (Hercules, CA, USA). Recombinant cytokines of TGF- $\beta 1$ and RANKL were from R\&D Systems (Minneapolis, MN, USA) and Pepro Tech (Rocky Hill, NJ, USA) respectively. TRAP staining kit was obtained from Sigma-Aldrich (St. Louis, MO, USA). The OSCC cell line of SCC25 was obtained from American Type Tissue Collection (ATCC, Rockvile, MD, USA), maintained in DMEM with $10 \%$ FBS and antibiotics (100 U/ml of penicillin $\mathrm{G}$ and $100 \mathrm{mg} / \mathrm{ml}$ of streptomycin) at $37^{\circ} \mathrm{C}$ in an incubator $\left(5 \% \mathrm{CO}_{2} / 20 \% \mathrm{O}_{2}\right)$. The murine macrophage cell line of RAW 264.7 was kindly given by Dr Hongwei Jiang from Sun yat-sen University. RAW 264.7 cells were cultured in $\alpha$-MEM with $10 \% \mathrm{FBS}$ at $37^{\circ} \mathrm{C}$ in a humidified $5 \% \mathrm{CO}_{2}$ atmosphere.

Immunohistochemistry. OSCC tissue from 10 patients with bone invasion was examined to validate the protein expression of E-cad, Vim and Snaill. Informed consent was obtained from each patient. Serial paraffin tissue sections $(5 \mu \mathrm{m})$ were dewaxed, rehydrated and treated with $0.3 \%$ hydrogen peroxiode in PBS. Antigen retrieval was performed by heating sections in a microwave oven $(2 \times 4 \mathrm{~min})$ with $0.2 \%$ citrate buffer $(\mathrm{pH}=6)$. After non-specific binding was blocked with 5\% BSA in PBS for $30 \mathrm{~min}$, sections were incubated with primary antibody of E-cad (1:100), Vim (1:100), Snail1 (1:200) overnight at $4^{\circ} \mathrm{C}$. Sections were then treated with anti-mouse/rabbit secondary antibodies (Envision+Systems) (Dako, Carpinteria, CA, USA) for $30 \mathrm{~min}$, followed by diaminobenzidine (DAB) detection solution for a few minutes at room temperature. Primary antibodies were replaced by non-immune serum as negative control. Sections were counterstained with Mayer's haematoxylin, dehydrated and mounted with mounting medium. The final results were visualized by light microscopy and photographed using a digital camera. Immunostaining intensity was scored according to the percentage of tumour cells positively-stained: and designated as + where $20 \%$ of cells were stained; ++ where $40 \%$ were stained; +++ where $60 \%$ were stained.

In vivo animal model of bone invasion by OSCC. Balb-c nude mice were purchased from the animal resources center, housed in animal facility, and cared for by animal house staff. All protocols were reviewed and approved by university ethics committee (2016-334QX). At 6-7 weeks old, these mice were utilized to develop an animal model of bone invasion by OSCC (3). Under sterile condition, OSCC cells of SCC25 $\left(5 \times 10^{6} / 100 \mu \mathrm{l}\right)$ were injected subcutaneously overlaying the calvaria. Mice were randomly divided into 2 groups ( $n=6 /$ group): the negative control group received PBS (Group 1: PBS); the positive control group received cells of SCC25 (Group 2: SCC25). Body weight and tumour volume were recorded every week. All animals were sacrificed at week 6 .

Micro-computed tomography $(\mu C T)$ imaging. Calvariae were surgically removed from PBS treated control, SCC25 tumours bearing nude mice, fixed in $70 \%$ ethanol and scanned by using a $\mu \mathrm{CT}$ instrument (Scanco Medical AG, Bassersdorf, Switzerland). $\mu \mathrm{CT}$-analyzer software was used to analyze the structure of calvaria with the global segmentation method. Two-dimensional images were used to generate three-dimensional reconstruction. The area of each calvaria was outlined for analysis and quantification.

Histological and immunohistochemical analysis. To perform histological analysis for the tumour-bearing calvaria, all calvariae were firstly decalcified with $10 \%$ EDTA ( $\mathrm{pH} 7.4)$ for 2 weeks and then processed for paraffin embedding. Serial $5-\mu \mathrm{m}$ sections of paraffin-embedded calvariae were stained by both hematoxylin and eosin $(\mathrm{H} \& \mathrm{E})$ and tartrate-resistant acid phosphatase (TRAP). Analysis of TRAP-positive osteoclast numbers at the tumour-bone interface was performed (3). For each section, an area of $2 \mathrm{~mm}^{2}$ with the tumour-bone interface was defined for counting osteoclast numbers. Four fields of this area were randomly selected and counted to determine the numbers of TRAP-postitive osteoclasts.

Immunohistochemial staining of sections was performed by incubation of serial sections with the primary antibody of E-cad (1:100), Vim (1:100), Snail (1:200) overnight, followed 
by HRP labeled secondary antibody and DAB staining. Specimens treated with non-immune serum served as negative controls. Immunostaining intensity was scored as above.

Cell proliferation assay. SCC25 cells were seeded at a density of $5 \times 10^{3}$ cells/well in 96 -well plates, allowed to attach overnight and then treated with TGF- $\beta 1(5 \mathrm{ng} / \mathrm{ml})$ for 2,4 and 6 days. Absorbance was read at $590 \mathrm{~nm}$ on a Biomek plate reader, after addition of $20 \mu 1$ methylthiazol tetrazolium (MTT, $5 \mathrm{mg} / \mathrm{ml}$, Life Technologies, Carlsbad, CA, USA) to the wells for $4 \mathrm{~h}$ of incubation, followed by removal of the solution and addition of $150 \mu \mathrm{l} /$ well of dimethyl suphoxide (DMSO, Sigma-Aldrich) to solubilize the cells.

Cell apoptosis assay. The apoptosis analysis of SCC25 cells with or without TGF- $\beta 1$ treatment was performed by flow cytometry analysis (FACS, Beckman Coulter, Brea, CA, USA), with the staining of Annexin V/PI (Life Technologies) based on the standard protocol. Cells were washed in ice-cold PBS at least three times, re-suspended in $100 \mu \mathrm{l}$ of binding buffer and incubated with Annexin V-fluorescein isothiocyanate for $15 \mathrm{~min}$ at $4^{\circ} \mathrm{C}$ in the dark. Cells were then incubated for $5 \mathrm{~min}$ with PI and analyzed by FACS.

Immunocytochemistry. After the treatment with TGF- $\beta 1$ for 2, 4 and 6 days, SCC25 cells were fixed with $70 \%$ ethanol for $10 \mathrm{~min}$ and permeabilized by $0.1 \%$ Triton X-100 for $5 \mathrm{~min}$. Non-specific binding of the antibodies was avoided by blocking with 5\% BSA in PBS for $30 \mathrm{~min}$, followed by incubation with primary antibodies of CK (1:200) and Vim (1:100) overnight at $4^{\circ} \mathrm{C}$, and then with secondary antibodies for $1 \mathrm{~h}$ at $37^{\circ} \mathrm{C}$. Non-immune serum instead of the primary antibody was used as negative control. Sites of binding were visualized using liquid diaminobenzidine (DAB) substrate chromogen system (Dako), counterstained with Mayer's haematoxylin, and photographed by a digital camera. Immunostaining intensity was scored as above.

Real-time PCR. Total RNA was isolated from SCC25 cells before and after TGF- $\beta 1$ treatment using PureLink RNA mini kit (Invitrogen, Carlsbad, CA, USA), and reverse transcribed to cDNA using iScript cDNA Synthesis kit (Bio-Rad) based on the manufacturer's instructions. Quantitative gene analysis was performed for E-cad, N-cad, Snaill and Vim by Express SYBR GreenER qPCR Supermix Universal kit (Invitrogen) and icycler iQ5 Real-time PCR system (Bio-Rad). The data were normalized to the internal control, GAPDH to obtain $\Delta \mathrm{Cq}$. Finally fold-change of genes of interest relative to untreated samples was reported by $2^{-\Delta \Delta C q}$ method (11). Primers used in this study are as reported elsewhere $(7,9,10)$.

Western blotting. Total protein was extracted from SCC 25 cells before and after TGF- $\beta 1$ treatment using lysis buffer (Thermo Fisher Scientific). The protein concentration was determined by a BCA Protein assay kit (Pierce, Rockford, IL, USA), and $40 \mu \mathrm{g}$ of protein was subjected to SDS-PAGE with $10 \%$ poly-acrylamide gels. Proteins were transferred to PVDF membranes, and blocked with 5\% non-fat dry milk in Tris-buffered saline (TBS) for $1 \mathrm{~h}$ at room temperature. The membranes were then incubated with primary antibodies of
E-cad (1:200), N-cad (1:200), Snail1 (1:200), Vim (1:100) and $\alpha$-tublin (1:3000) overnight at $4^{\circ} \mathrm{C}$, washed twice and incubated with horseradish peroxidase-conjugated (HRP) secondary antibodies for $1 \mathrm{~h}$ at room temperature. The protein bands were detected by SuperSignal WestPico Chemiluminescent Substrate (Thermo Fisher Scientific) and visualized using VersaDoc-MP Imaging Systems (Bio-Rad).

Indirect cell co-culture. Transwell inserts $(0.4-\mu \mathrm{m}$ pore, Corning, Inc., Corning, NY, USA) were used in the indirect cell co-culture. SCC 25 cells $\left(5 \times 10^{3}\right.$ cells/well) were seeded in the upper chamber with the treatment of TGF- $\beta 1(5 \mathrm{ng} / \mathrm{ml})$, and RAW 264.7 cells $\left(5 \times 10^{4}\right.$ cells/well) were placed in the 24-well plates. The chambers were incubated for 2, 4 and 6 days. To examine the effects of TGF- $\beta 1$, total RNA were extracted from SCC25 and RAW 264.7 on each time point and reverse transcribed to cDNA. Quantitative gene analysis of E-cad, Snaill, RANKL for SCC25, and E-cad, TRAP, nuclear factor of activated T-cells cytoplasmic 1 (NFATc1) for RAW 264.7 was performed by SYBR Green ER qPCR Supermix (Invitrogen) and icycler iQ5 Real-time PCR system (Bio-Rad). Changes of gene expression were analyzed as above.

Immunofluorescence. To further confirm the switch of E-cad protein, immunofluorescence (IF) was utilized. Cells of SCC25 and RAW 264.7 were placed into transwell inserts and treated as above. On each time point, both cells of SCC25 and RAW 264.7 were fixed with $75 \%$ ethanol for $10 \mathrm{~min}$, and blocked in 5\% bovine serum albumin for $30 \mathrm{~min}$. Primary antibody of E-cad (1:50) were incubated at $4^{\circ} \mathrm{C}$ overnight, followed by detection with fluorescence-conjugated secondary antibody (FITC, 1:200, Boster) at room temperature for $2 \mathrm{~h}$. DAPI staining (Boster) was used to visualize the nuclei. Images were acquired and photographed by a Nikon inverted microscope. Normal serum replaced primary antibodies as negative controls.

Osteoclast differentiation from RAW 264.7 cells. Cells of SCC25 and RAW 264.7 were placed into the transwell inserts, treated as above. To obtain osteoclasts, RAW 264.7 were supplemented with $50 \mathrm{ng} / \mathrm{ml}$ of recombinant mouse RANKL (Pepro Tech). Medium change and new cytokine were added every 2 days, while osteoclasts appeared after 4 days of treatment. These osteoclasts were subsequently fixed in $10 \%$ formalin. TRAP staining and IF of F-actin staining were used to characterize osteoclasts. TRAP positive cells of three or more nuclei were considered to be multinucleated osteoclasts. Rhodamine-conjugated phalloidin (Life Technologies) were used to stain F-actin. DAPI staining (Boster) was used to visualize the nuclei. Four fields were randomly selected and counted for osteoclast and F-actin numbers.

Statistical analysis. Results were presented as mean \pm standard error $(\mathrm{M} \pm \mathrm{SE})$ of at least 3 independent experiments. Data analysis was performed using SPSS software (Version 20.0, IBM, USA). Student's t-test was used to compare two means. One way analysis of variance was applied to compare two or more means, followed by Student-Newan-Keulls test. A p-value of $<0.05$ was regarded as statistically significant. 


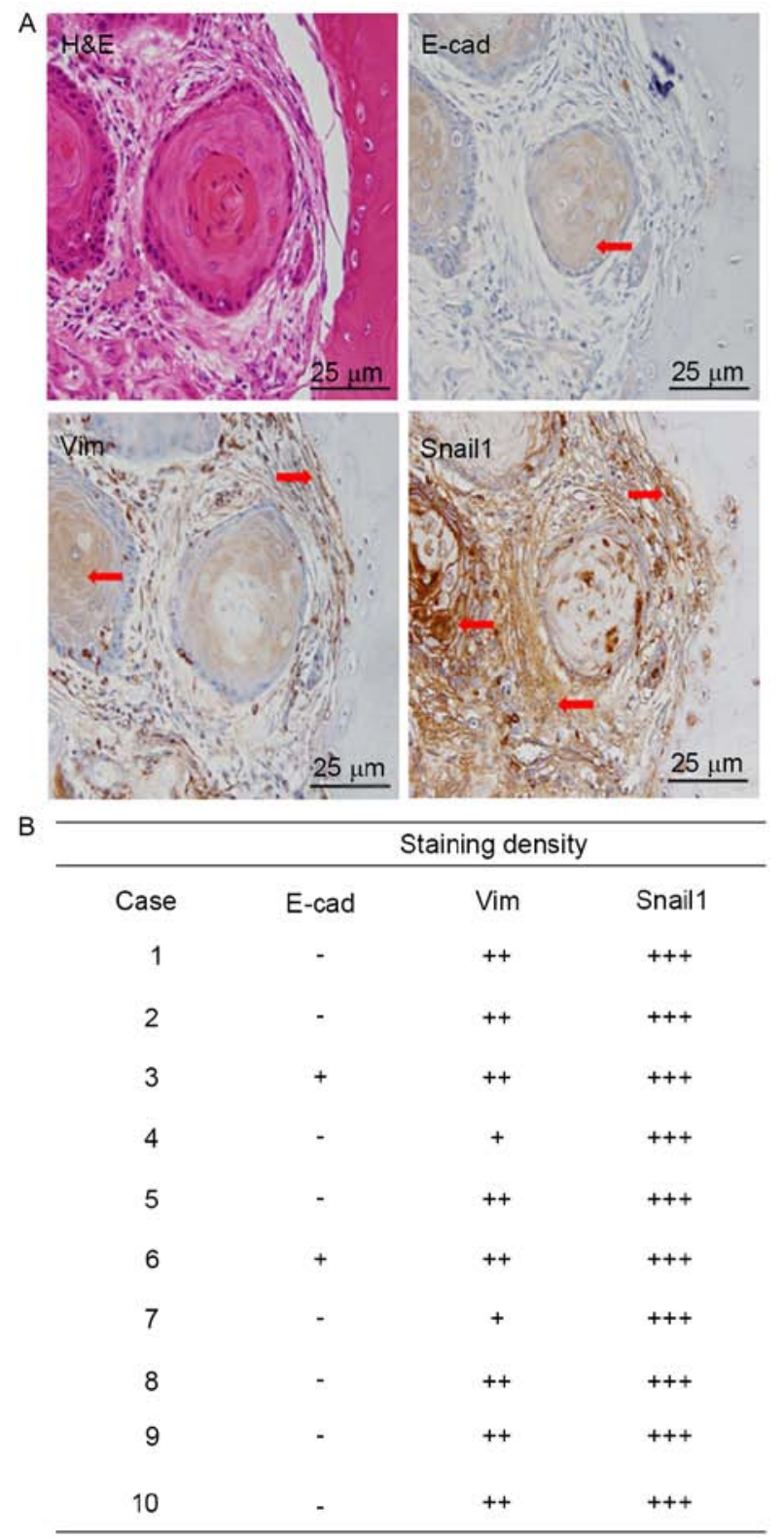

Figure 1. Results of IHC from OSCC tissue samples with bone invasion. (A) Weak staining of E-cad protein was found in cytoplasm of tumour cells, moderate staining of Vim and strong staining of Snaill was observed in cell cytoplasm (arrow, DAB, bar, $25 \mu \mathrm{m}$ ). (B) A summary of staining density of all clinical samples.

\section{Results}

Weak staining of E-cad protein is observed in tissue samples from OSCC patients with bone invasion. OSCC tissue samples with bone invasion were obtained from 10 patients and used for IHC. Results showed weak staining of E-cad protein was found in cytoplasm of tumour cells, moderate staining of Vim and strong staining of Snail1 was observed in cell cytoplasm (Fig. 1A). A summary of staining density was shown in Fig. 1B.

Animal model of OSCC with bone invasion established by using SCC 25 cells. The animal model of OSCC with bone invasion was established by using SCC25 cells injected through the central area of calvariae (Fig. 2A). No significant differences of body weight were found between PBS control
A
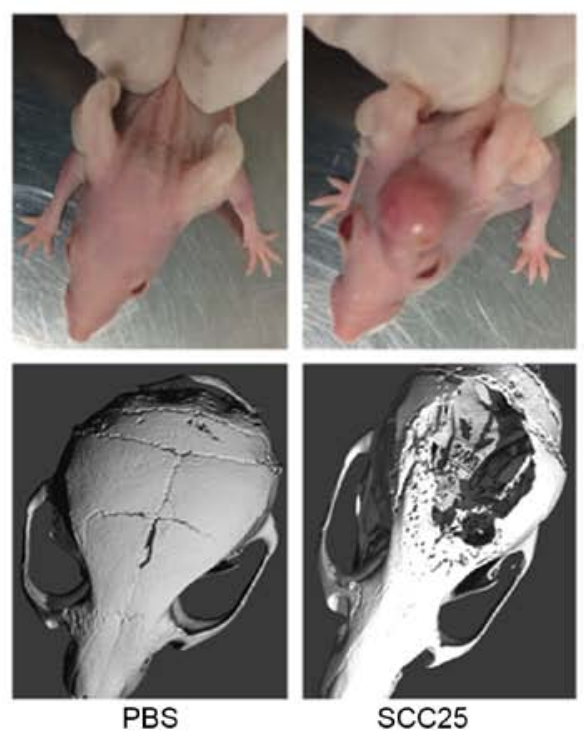

B
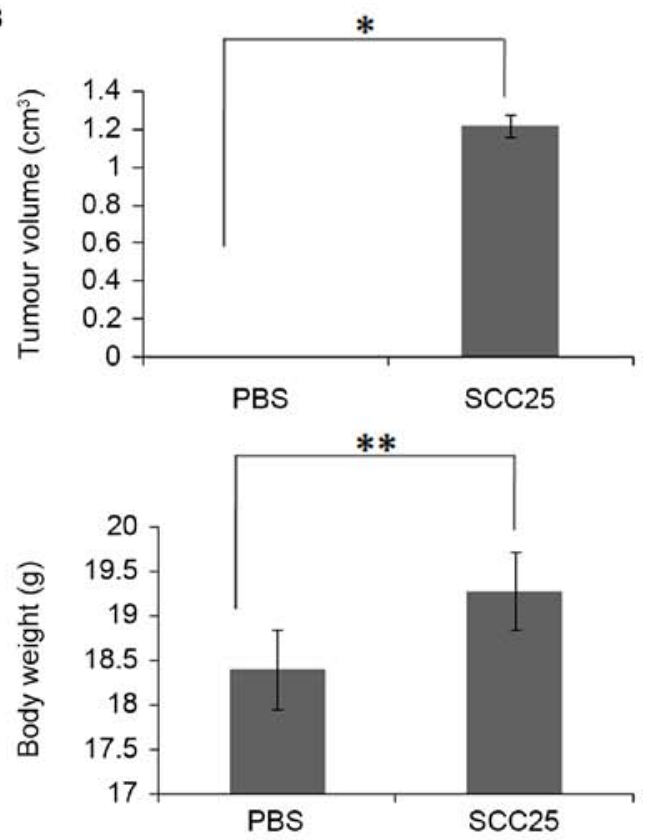

Figure 2. The animal model of OSCC with bone invasion was established by SCC 25 cells. (A) $\mu \mathrm{CT}$ analysis showed typical bone invasion in SCC25 group, while PBS control group had not formed a tumour. (B) No significant differences of body weight was found between groups of PBS control and $\operatorname{SCC} 25\left({ }^{*} \mathrm{p}<0.05 ;{ }^{* *} \mathrm{p}>0.05\right)$.

group and SCC25 group (Fig. 2B). $\mu \mathrm{CT}$ imaging found typical bone invasion of SCC25 group, while the control group had not formed a tumour (Fig. 2A and B). Histological analysis showed plenty of osteoclasts accumulating in the tumour-bone interface, while less osteoclasts were observed in the bone marrow of the control group (Fig. 3A and B).

Validation of the E-cad protein in animal tissue samples with bone invasion. IHC of animal tissue samples further confirmed the loss of E-cad protein. Results detected less staining of E-cad protein was found in cytoplasm of tumour cells, with moderate staining of Vim and strong staining of Snaill (Fig. 4A). A summary of staining results was supplied in Fig. 4B. 


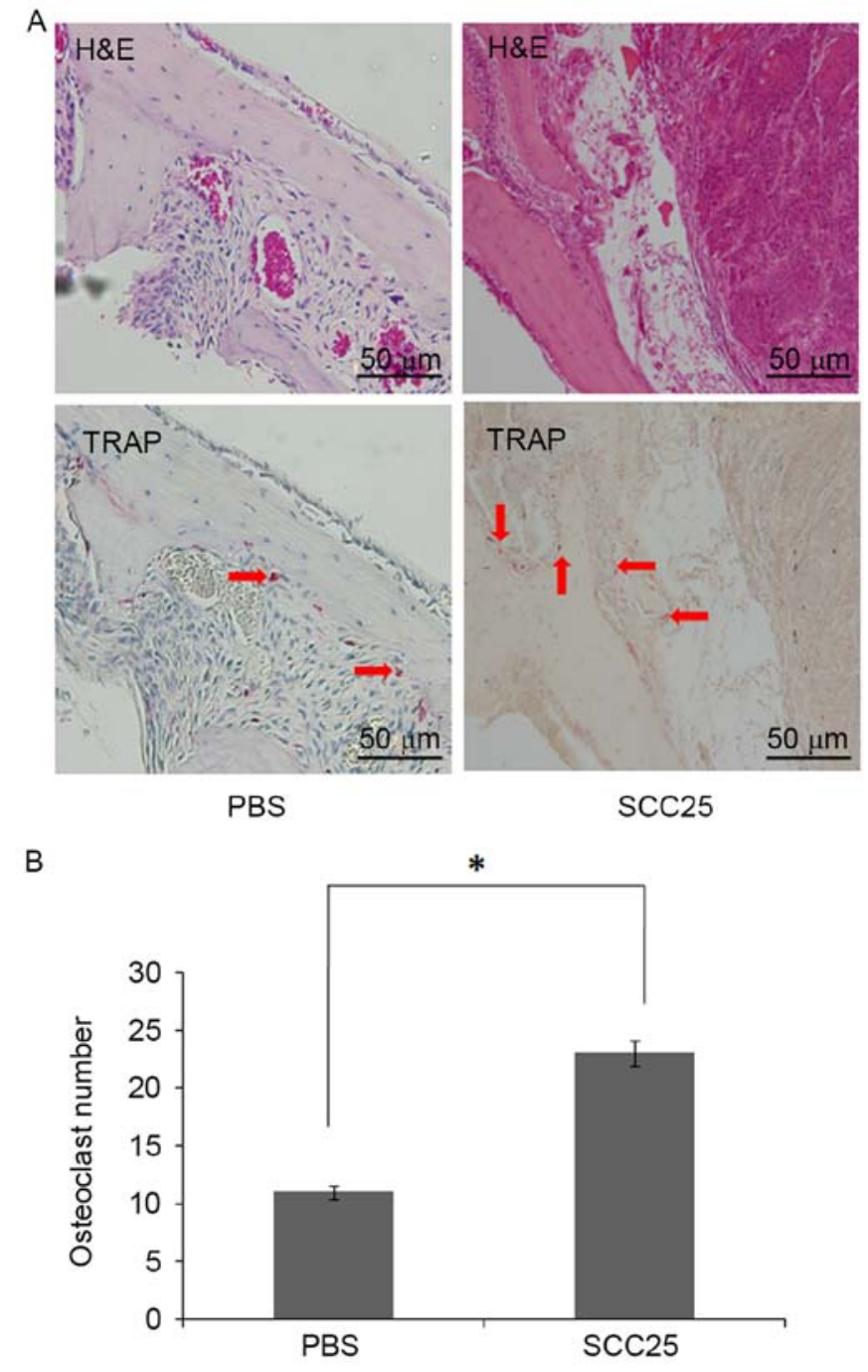

Figure 3. Histological analysis of animal tissues with bone invasion. (A) Results showed plenty of osteoclasts accumulating in the tumour-bone interface, while less osteoclasts were observed in the bone marrow tissue of control group (arrow, TRAP, bar, $50 \mu \mathrm{m}$ ). (B) Quantification of the osteoclast number $(* \mathrm{p}<0.05)$.

TGF- $\beta 1$ has no inhibitory effect on proliferation and apoptosis of SCC 25 cells. MTT assay demonstrated that $5 \mathrm{ng} / \mathrm{ml}$ of TGF- $\beta 1$ had no inhibitory effect on the proliferation of SCC25 cells during 6 days of treatment (Fig. 5A). FACS analysis showed the apoptotic subpopulation of SCC25 cells with TGF- $\beta 1$ treatment was similar with that of normal SCC25 cells (Fig. 5B).

Artificial EMT is induced in SCC25 cells with TGF- $\beta 1$ treatment. Since TGF- $\beta 1$ could induce the artificial EMT, $5 \mathrm{ng} / \mathrm{ml}$ of TGF- $\beta 1$ was used to treat SCC 25 cells for 2,4 and 6 days. The cell morphology was not obviously changed, still slab stone cell shape (Fig. 5C). IHC found CK staining remained the same before and after TGF- $\beta 1$ treatment, and slight staining of Vim was observed in cytoplasm of SCC25 cells (Fig. 5C and D). Real-time PCR was utilized to examine mRNA expression of selected EMT markers: E-cad decreased while N-cad increased, Snaill decreased then increased while Vim increased (Fig. 6A). Western blotting further confirmed these EMT marker changes at the protein level (Fig. 6B).
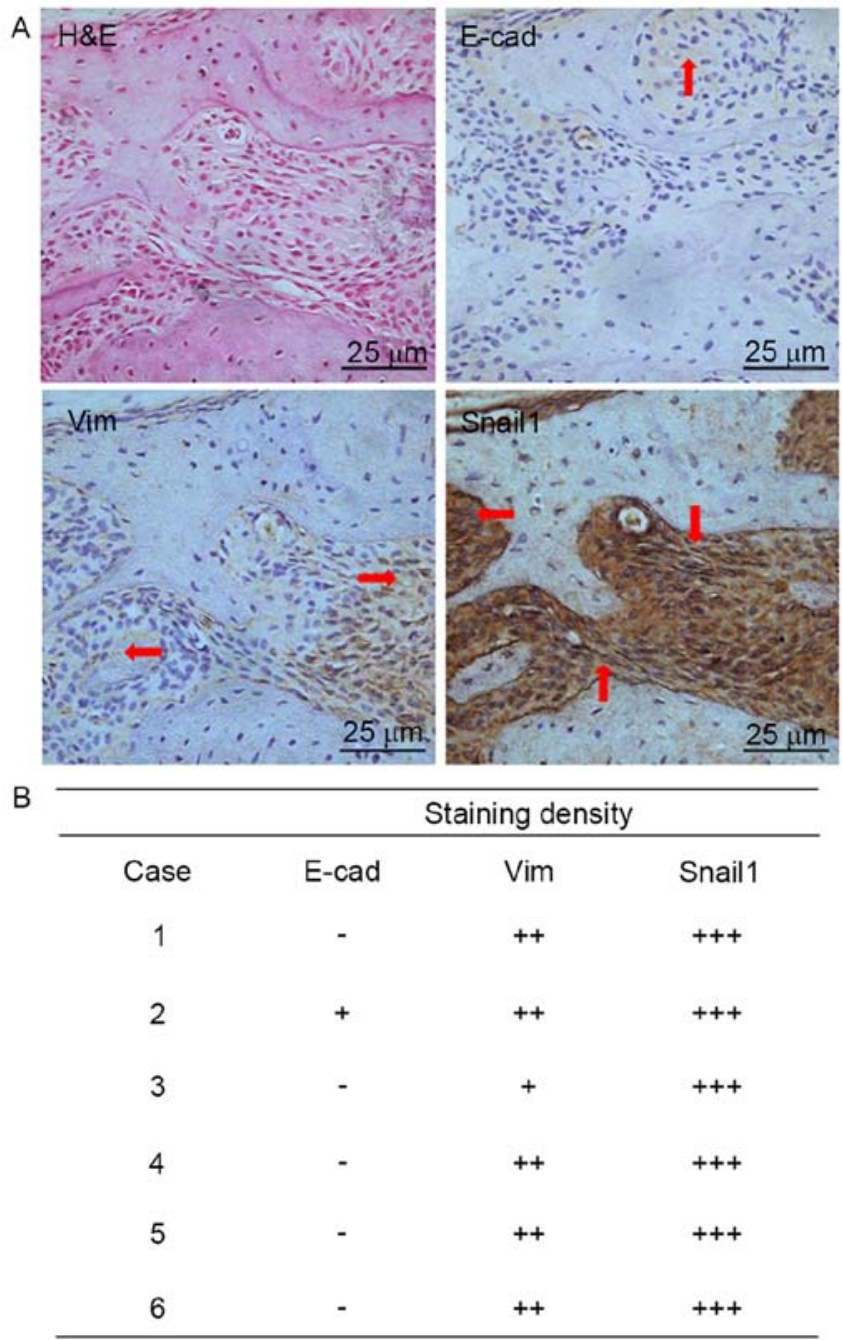

Figure 4. Validation of the E-cad protein in animal tissue samples. (A) Results of IHC showed less staining of E-cad protein was found in cytoplasm of tumour cells, moderate staining of Vim and strong staining of Snaill was found in cell cytoplasm (arrow, DAB, bar, $25 \mu \mathrm{m}$ ). (B) A summary of staining results.

The indirect co-culture model is utilized in cells of SCC25 and RAW 264.7. The indirect cell co-culture of SCC25 and RAW 264.7 was performed with 2, 4 and 6 days, and on each time point both cells were obtained to extract RNA and real-time PCR was performed. Results showed that E-cad mRNA decreased in SCC25 cells on day 4 and day 6, while Snaill and RANKL increased (Fig. 7A). Conversely, E-cad mRNA increased in RAW 264.7 cells on day 4 and day 6 , while TRAP and NFATc1 increased from day 4 to day 6 (Fig. 7A).

Switch of E-cad staining is observed in SCC25 and RAW 264.7 cells. To further confirm the changes of E-cad protein in the indirect co-culture model, SCC25 and RAW 264.7 cells were stained with E-cad and observed by IF. Results indicated a switch change of E-cad staining in both cell types: the staining of E-cad was observed to decrease in SCC25 cells, while increase in RAW 264.7 cells from day 4 to day 6 during the cell co-cultures (Fig. 7B).

More osteoclasts are generated from RAW 264.7 cells with RANKL treatment. With the increase of E-cad in RAW 264.7 
A

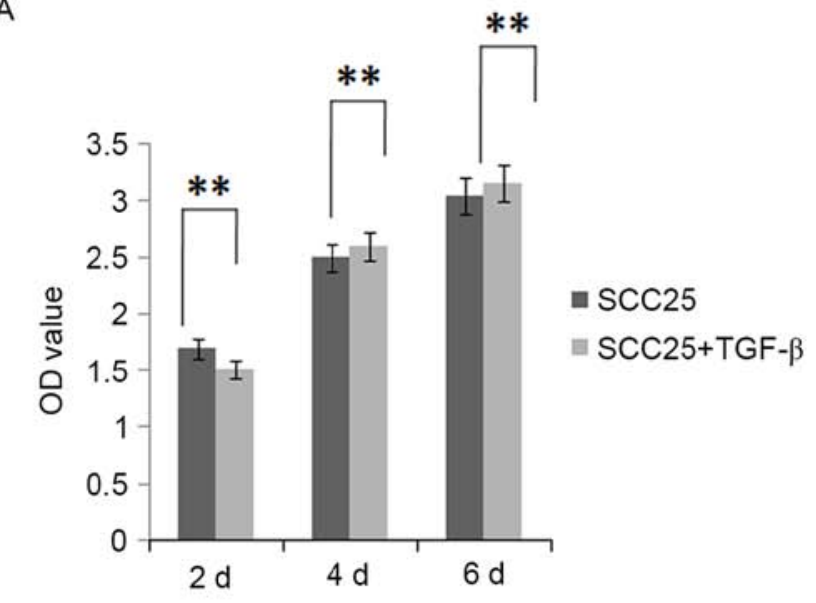

B

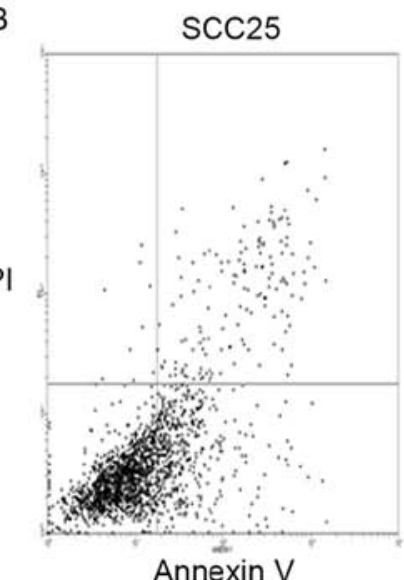

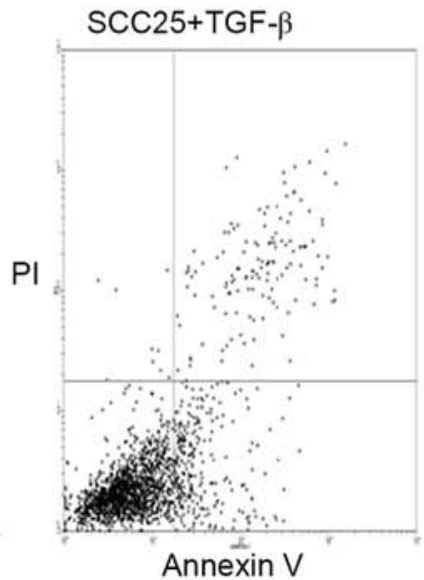

C

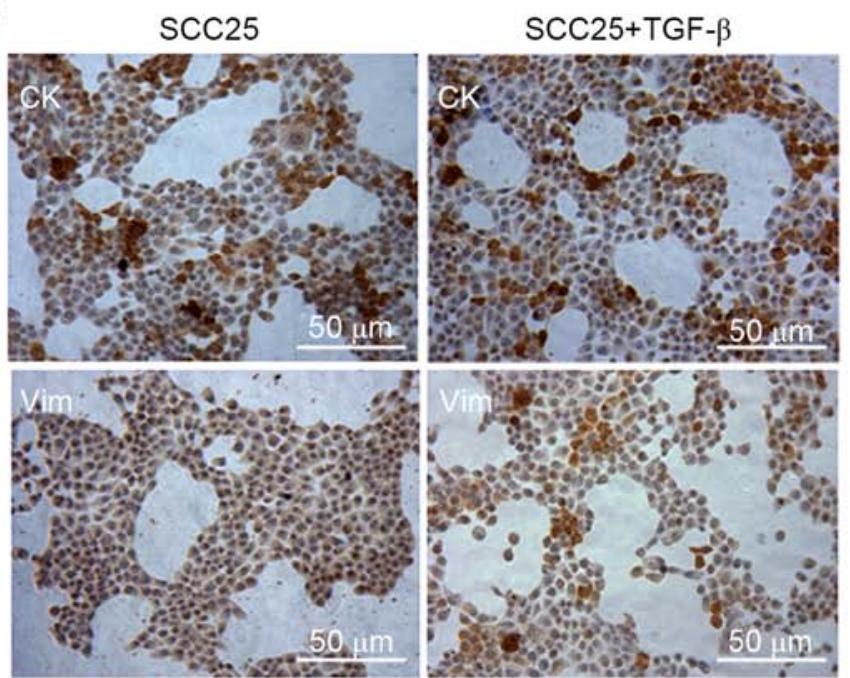

D
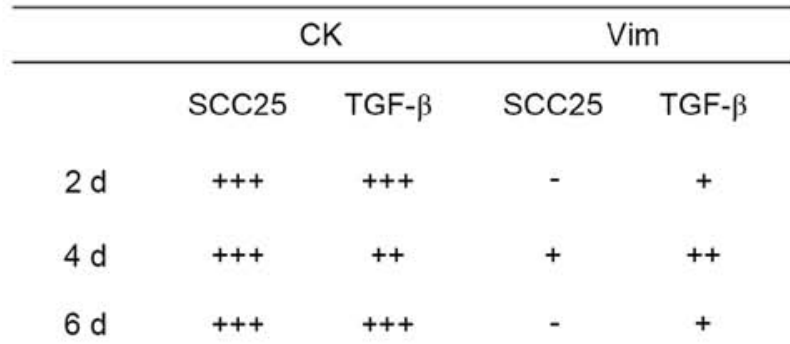

Figure 5. Effects of TGF- $\beta 1$ on SCC25 cells. (A) MTT assay demonstrated that $5 \mathrm{ng} / \mathrm{ml}$ of TGF- $\beta 1$ had no inhibitory effects on proliferation of SCC 25 cells ( $*$ p $>0.05$ ). (B) FACS analysis suggested TGF- $\beta 1$ had no apoptotic effects with SCC 25 cells. (C) The cell morphology was not obviously changed (still slab stone cell shape). IHC found CK staining was the same before and after TGF- $\beta 1$ treatment, and slight staining of Vim was found in cytoplasm (DAB, bar, $50 \mu \mathrm{m}$ ). (D) A summary of staining density results.

cells, next we asked whether more osteoclasts would differentiate with the supplement of RANKL. We still used the indirect cell co-culture and added the cytokine of RANKL within RAW 264.7 cells. Results of TRAP staining showed some giant cells on day 2 , while more osteoclasts differentiated on day 4 , and a maximum number of osteoclasts was found on day 6 (Fig. 8A and B). The F-actin staining suggested the same trend with TRAP staining, and more staining of F-actin was observed in osteoclasts on day 6 by IF (Fig. 8A and B).

\section{Discussion}

Cadherins, involved in cell-cell contact, are regulated trans-membrane calcium-dependent proteins. One of the main cadherins is E-cad, which is responsible for intercellular adhesion between epithelial cells (12). For several decades, E-cad has been reported as a major constituent of adherens junction, mediating adhesion between epithelial cells, therefore safeguarding epithelial barrier integrity (13). If carcinogens induce the ligation of epithelial layer, E-cad would no longer work and maintain the normal function. The neoplastic cells at the invasive tumour front lose their epithelial cell phenotype and acquire mesenchymal-like phenotype referred to as EMT. The loss of E-cad and the acquisition of $\mathrm{N}$-cad, so-called 'cadherin switch', is regarded as a hallmark of EMT (8).

Except for epithelial cells, recent progress has uncovered a critical role of E-cad in mononuclear phagocyte function. It becomes increasingly clear that E-cad and its associated catenins are expressed in dendritic cells, langerhans cells, macrophages and osteoclasts (13). For example, interleukin-4 (IL-4) stimulated macrophages, which fuse to become multinucleated foreign body giant cells, increase expression of E-cad protein (14). Additionally, multiple studies have implicated $\mathrm{M}$ - and $\mathrm{N}$-cad during myoblast fusion and differentiation (15). As for osteoclasts, Mbalaviele et al suggested that E-cad participated in the resorptive function (16). The study by Fiorino and Harrison demonstrated a role for E-cad-based signaling of osteoclast differentiation (10).

With E-cad and its related reports on osteoclasts, we re-considered our research focus, since bone invasion by OSCC is crosstalk between osteoblasts, osteoclasts, and tumour cells. Especially, the loss of E-cad protein is observed in OSCC tissue samples from patients with bone invasion. This was confirmed in our previous reports (9) and our current research. In the present study, we also constructed an animal 
model of OSCC with bone invasion by using SCC25 cells. After 6 weeks this model was successfully established, and histological analysis also found similar changes of E-cad protein, which further confirm our hypothesis that loss of E-cad may be utilized by osteoclast precursors. We perform double staining of E-cad and TRAP to locate osteoclasts, but we did not get any staining of E-cad on osteoclasts. We considered that we could not get time course window on human tissue samples to capture the switch of E-cad protein. What we observed are the terminal stage of mature osteoclasts, which could not mimic the procedure how E-cad moves from tumour cells to osteoclast precursors. Therefore, we used the indirect cell co-culture model to further explore our hypothesis.

To induce the loss of E-cad in OSCC cells, we utilized TGF- $\beta 1$ as the inducer since TGF has long been reported to be a key initiator of EMT. EMT process can be categorized into three well-defined sub-types and TGF- $\beta$ are involved in all three types (5). Type 1 EMT is known as developmental EMT and disruption of TGF- $\beta$ isoforms and their receptors has been associated with defects in type 1 EMT. Type 2 EMT is induced in response to inflammation, particularly wound healing and tissue regeneration. TGF- $\beta$ plays an instrumental role in mediating this process. Type 3 EMT is most prevalent for oncogenically transformed cells which are capable of metastasizing. Studies of in vitro suggest a major role of TGF- $\beta$ signaling in the induction of EMT in cancer cells.

By using TGF- $\beta 1$ in the current study, we confirm the artificial EMT of SCC25 cells, with EMT marker changes, and slight cell phenotype changes. This is the basis of simulation, and it is possible to check whether E-cad would be utilized by RAW 264.7 cells only if E-cad deceases in SCC25 cells. Since TGF- $\beta 1$ has no inhibitory effects on RAW 264.7 cells, we used the indirect cell co-culture model to check whether there are changes of E-cad between these two cell types. Real-time PCR showed E-cad mRNA increased in monocytes and IF found the obvious switch of E-cad staining from SCC25 to RAW 264.7. This may be explained by two potential mechanisms. On the one hand, E-cad may be shaded by proteases such as matrix metalloproteinases (MMPs) and secreted into extracellular medium, which are further 'hijacked' by RAW 264.7 cells. On the other hand, whether TGF- $\beta 1$ has stimulative effects on E-cad expression of RAW 264.7 cells needs to be further investigated. Therefore, whether the switch of E-cad is the result of simple protein movement, or the innate response between two types of cells is the next research question to be addressed in the near future.

With the increased amount of E-cad in RAW 264.7 cells, we want to confirm if more osteoclasts would differentiate with the supplement of RANKL, which is the key transcriptional factor for osteoclast development (17). Actually, within the cell co-culture, real-time PCR detected increased expression of RANKL mRNA in SCC25 cells. Additionally, results of TRAP and F-actin staining confirmed the increased number of osteoclasts after adding the cytokine of RANKL. Being consistent with the report by Fiorino and Harrison (10), these results confirmed the important roles of E-cad in the early differentiation of osteoclasts and proved the key roles of RANKL in osteoclast maturation.

E-cad is not only the key component within cell adherens junction but also the starter of cellular signaling for bone cell

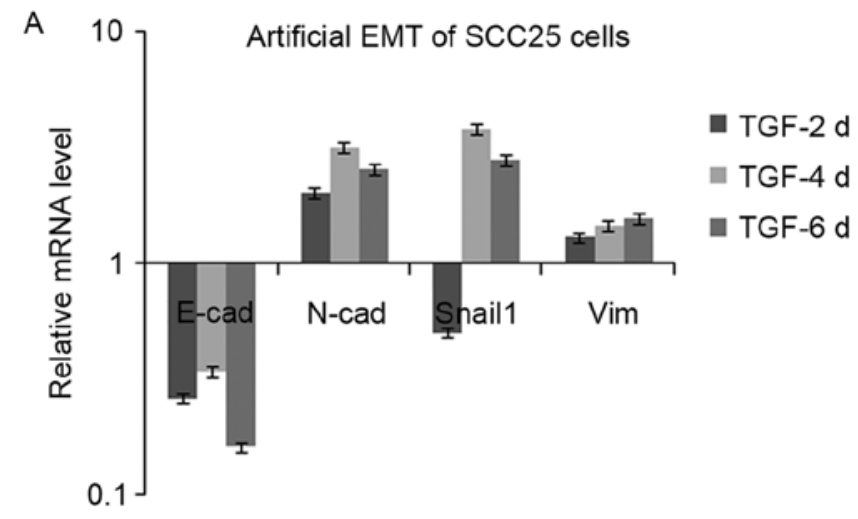

B
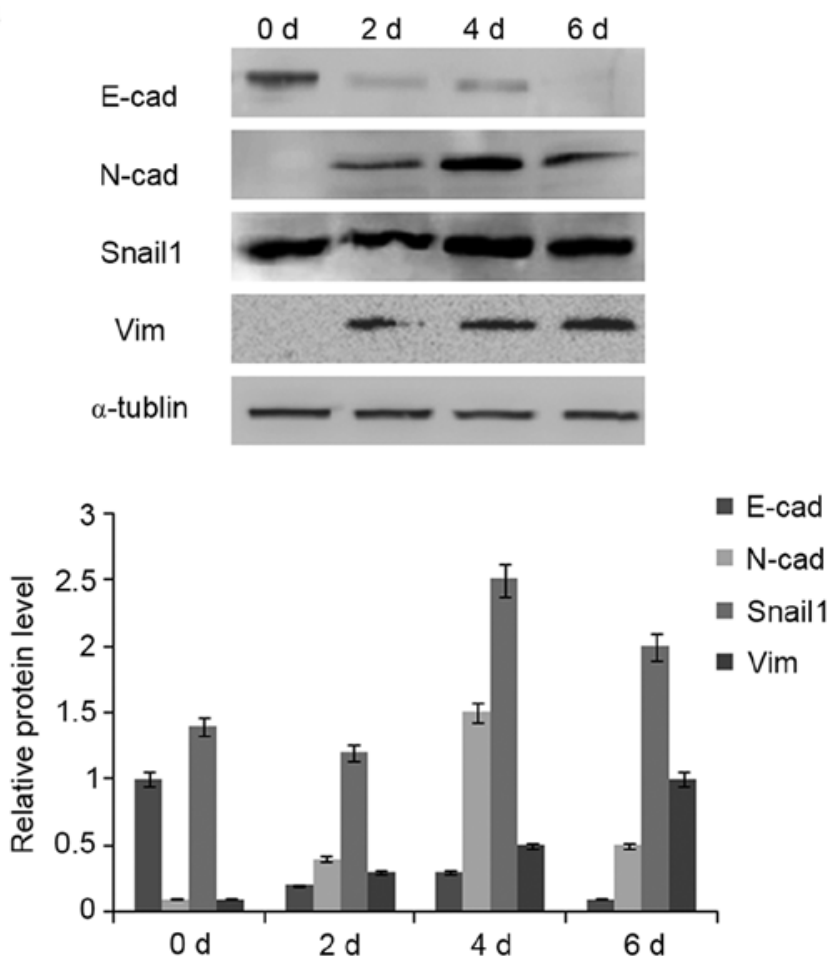

Figure 6. Artificial EMT of SCC25 cells induced by TGF- $\beta 1$. (A) Real-time PCR detected the mRNA expression changes of EMT markers: E-cad decreased while $\mathrm{N}$-cad increased on each time point, Snaill increased on day 4 and day 6, while Vim increased on each time point. (B) Western blotting further confirmed these changes at the protein level.

fate. As for osteoclastogenesis, a possible explanation of how E-cad works with other factors especially chemokines is that, the chemokines may recruit and mobilize monocytes first, and then E-cad connects them more tightly to fuse, which would easily differentiate into osteoclasts in a short time. These may supply therapeutic implications for blocking agent development in vivo, and specific peptides, antibodies or mutant proteins would be developed in an effort for potential clinical use (18). Nonetheless, the underling mechanisms have to be discovered through a variety of experiments in vitro.

Taken together, our study found the switch of E-cad protein was observed in the progression of bone invasion by OSCC. The loss of E-cad in tumour cells may be utilized by monocytes to differentiate into osteoclasts, further explaining the mechanisms of bone invasion by OSCC, and indicating that cross-talk between tumour cells, osteoblasts, 
A
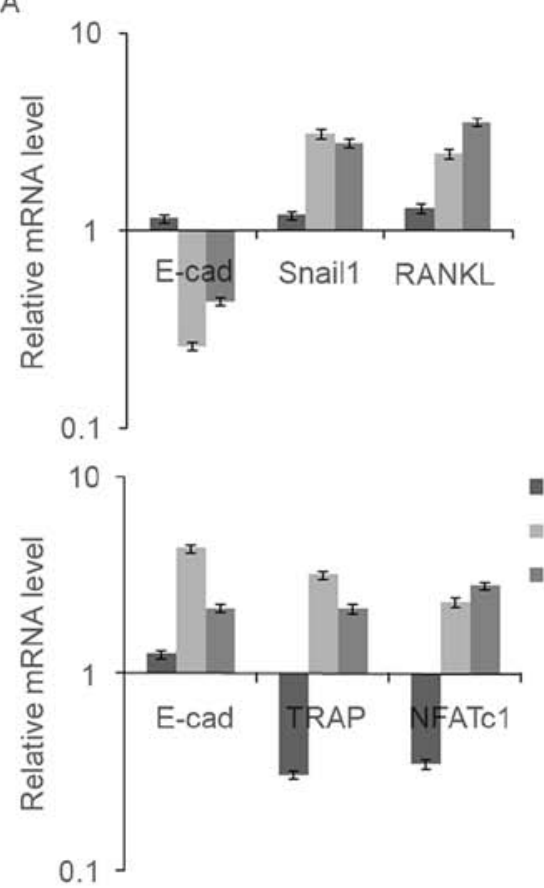

B

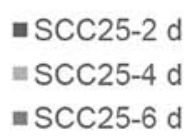

- SCC25-2 d

$=\operatorname{SCC} 25-4 \mathrm{~d}$

$\because \operatorname{scC} 25-6 \mathrm{~d}$
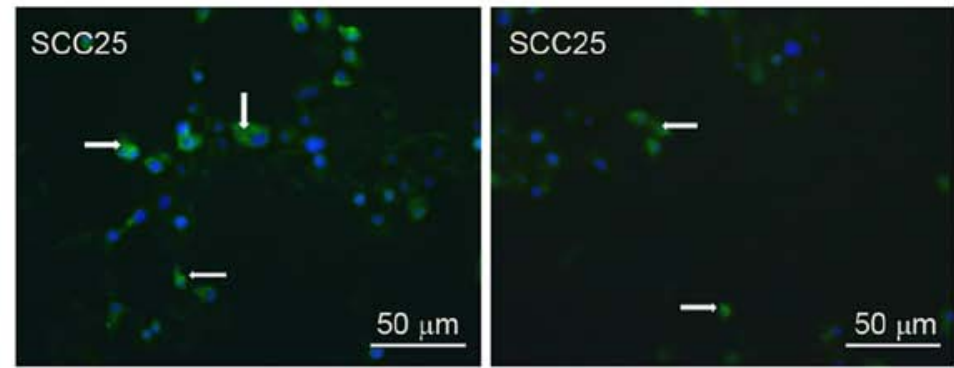

- RAW264.7-2 d

$=$ RAW264.7-4 d

= RAW264.7-6 d
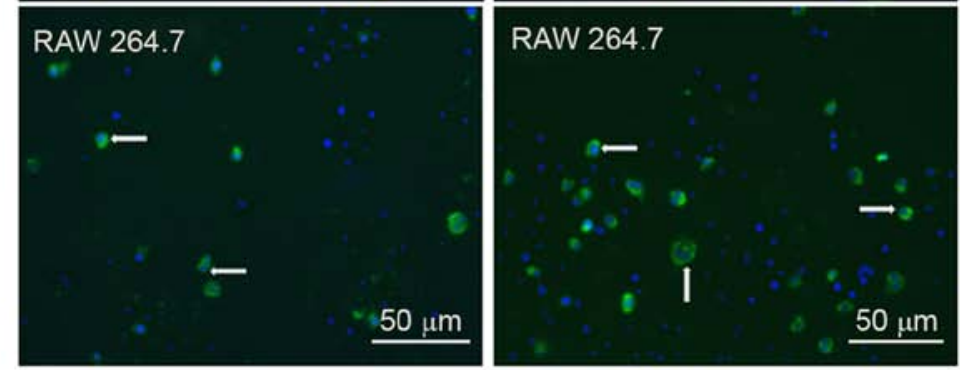

E-cad staining by IF

Figure 7. Indirect co-culture was utilized with cells of SCC25 and RAW 264.7. (A) The indirect cell co-culture was performed for 6 days, on each time point both cells were used to extract RNA and real-time PCR was performed. Results showed E-cad mRNA decreased in SCC25 cells on day 4 and day 6 , while Snail1 and RANKL increased. Conversely, E-cad mRNA increased in RAW 264.7 cells on day 4 and day 6, while TRAP and NFATc1 increased from day 4 to day 6. (B) To further confirm the changes of E-cad protein in the indirect co-culture, both cells of SCC25 and RAW 264.7 were stained with E-cad and observed by IF. Results indicated a switch of E-cad staining: the staining of E-cad was observed to decrease in SCC25 while increase in RAW 264.7 from day 4 to day 6 (arrow, FITC, bar, $50 \mu \mathrm{m}$ ).
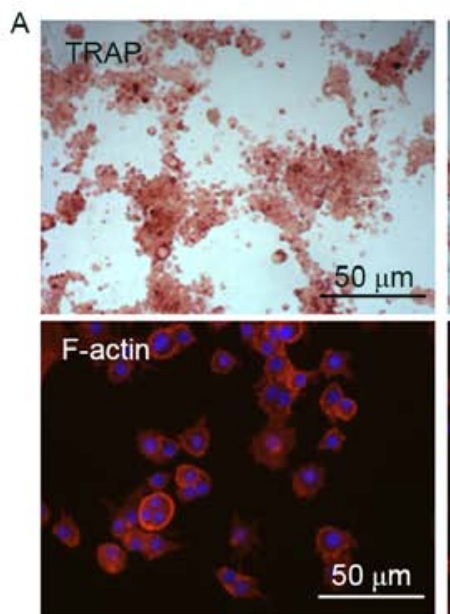

$0 d$

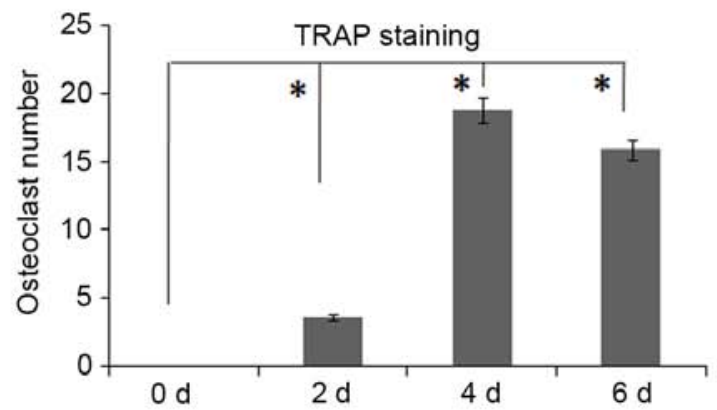

$2 d$
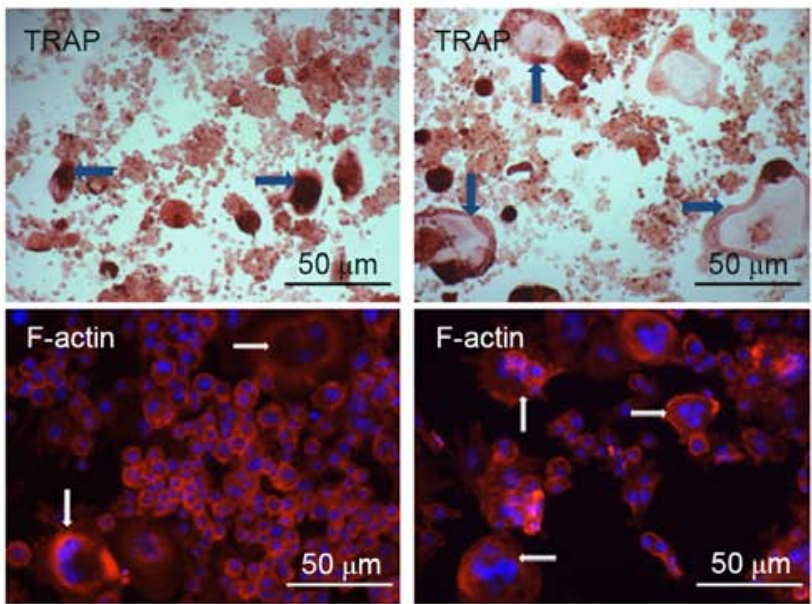

$4 d$

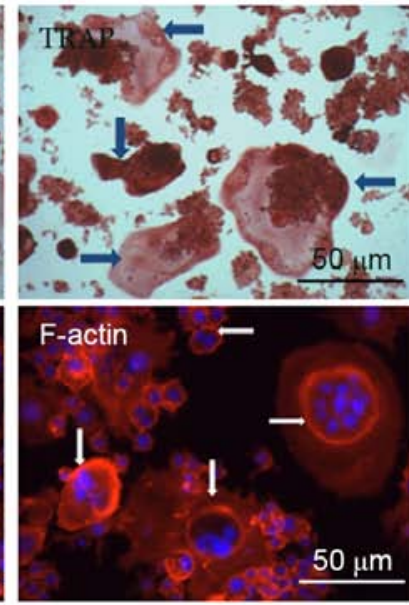

$6 d$

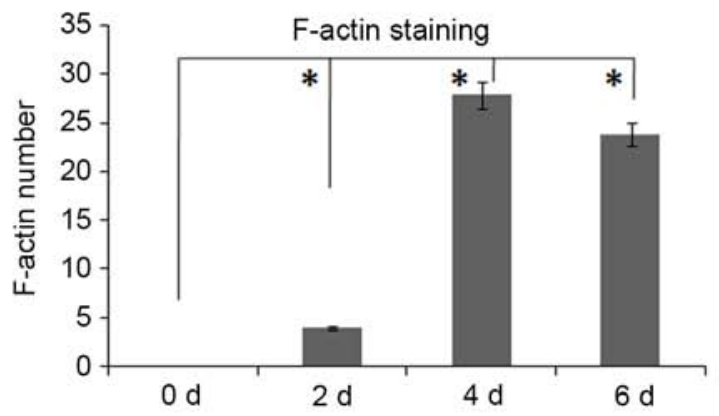

Figure 8. More osteoclasts are generated from RAW 264.7 cells with RANKL treatment. The indirect co-culture was used and the cytokine of RANKL was added to check whether more osteoclasts would differentiate. (A) TRAP staining showed some giant cells on day 2, while more osteoclasts differentiated on day 4 , and a maximum number of osteoclasts differentiated on day 6 (arrow, TRAP, bar, $50 \mu \mathrm{m}$ ). The F-actin staining suggested the same trend with TRAP staining, and more staining of F-actin was observed on day 6 by IF (arrow, TRITC, bar, $50 \mu \mathrm{m}$ ). (B) Quantification of osteoclast and F-actin numbers ( $\mathrm{p}<0.05$ ). 
and osteoclasts really exist, which may supply clues for future molecular biotherapies.

\section{Acknowledgements}

This study was supported by Foundation of Sun Yat-sen University (13ykpy41), Medical Scientific Research Foundation of Guangdong Province (B2014164) and National Natural Science Foundation of China (81500839).

\section{References}

1. Hwang YS, Ahn SY, Moon S, Zheng Z, Cha IH, Kim J and Zhang X: Insulin-like growth factor-II mRNA binding protein-3 and podoplanin expression are associated with bone invasion and prognosis in oral squamous cell carcinoma. Arch Oral Biol 69: 25-32, 2016.

2. Quan J, Zhou C, Johnson NW, Francis G, Dahlstrom JE and Gao J: Molecular pathways involved in crosstalk between cancer cells, osteoblasts and osteoclasts in the invasion of bone by oral squamous cell carcinoma. Pathology 44: 221-227, 2012.

3. Quan J, Morrison NA, Johnson NW and Gao J: MCP-1 as a potential target to inhibit the bone invasion by oral squamous cell carcinoma. J Cell Biochem 115: 1787-1798, 2014.

4. Quan J, Johnson NW, Zhou G, Parsons PG, Boyle GM and Gao J: Potential molecular targets for inhibiting bone invasion by oral squamous cell carcinoma: A review of mechanisms. Cancer Metastasis Rev 31: 209-219, 2012.

5. Syed V: TGF- $\beta$ signaling in cancer. J Cell Biochem 117: 1279-1287, 2016.

6. Nieto MA, Huang RY, Jackson RA and Thiery JP: EMT: 2016 Cell 166: 21-45, 2016.

7. Mani SA, Guo W, Liao MJ, Eaton EN, Ayyanan A, Zhou AY, Brooks M, Reinhard F, Zhang CC, Shipitsin M, et al: The epithelial-mesenchymal transition generates cells with properties of stem cells. Cell 133: 704-715, 2008.

8. Qiao B, Johnson NW and Gao J: Epithelial-mesenchymal transition in oral squamous cell carcinoma triggered by transforming growth factor-beta1 is Snail family-dependent and correlates with matrix metalloproteinase-2 and -9 expressions. Int J Oncol 37: $663-668,2010$
9. Quan J, Elhousiny M, Johnson NW and Gao J: Transforming growth factor- $\beta 1$ treatment of oral cancer induces epithelialmesenchymal transition and promotes bone invasion via enhanced activity of osteoclasts. Clin Exp Metastasis 30: 659-670, 2013.

10. Fiorino $\mathrm{C}$ and Harrison RE: E-cadherin is important for cell differentiation during osteoclastogenesis. Bone 86: 106-118, 2016.

11. Livak KJ and Schmittgen TD: Analysis of relative gene expression data using real-time quantitative PCR and the 2(- $-\Delta \Delta C(T))$ method. Methods 25: 402-408, 2001

12. Pereira CH, Morais MO, Martins AF, Soares MQ, Alencar RC, Batista AC, Leles CR and Mendonça EF: Expression of adhesion proteins (E-cadherin and $\beta$-catenin) and cell proliferation $(\mathrm{Ki}-67)$ at the invasive tumor front in conventional oral squamous cell and basaloid squamous cell carcinomas. Arch Oral Biol 61: 8-15, 2016.

13. Van den Bossche J, Malissen B, Mantovani A, De Baetselier P and Van Ginderachter JA: Regulation and function of the E-cadherin/catenin complex in cells of the monocyte-macrophage lineage and DCs. Blood 119: 1623-1633, 2012.

14. Moreno JL, Mikhailenko I, Tondravi MM and Keegan AD: IL-4 promotes the formation of multinucleated giant cells from macrophage precursors by a STAT6-dependent, homotypic mechanism: Contribution of E-cadherin. J Leukoc Biol 82: 1542-1553, 2007.

15. Gavard J, Marthiens V, Monnet $\mathrm{C}$, Lambert $\mathrm{M}$ and Mège RM: $\mathrm{N}$-cadherin activation substitutes for the cell contact control in cell cycle arrest and myogenic differentiation: Involvement of p120 and beta-catenin. J Biol Chem 279: 36795-36802, 2004.

16. Mbalaviele G, Chen H, Boyce BF, Mundy GR and Yoneda T: The role of cadherin in the generation of multinucleated osteoclasts from mononuclear precursors in murine marrow. J Clin Invest 95: 2757-2765, 1995.

17. Ikeda $\mathrm{K}$ and Takeshita S: The role of osteoclast differentiation and function in skeletal homeostasis. J Biochem 159: 1-8, 2016.

18. Marie PJ, Haÿ E and Saidak Z: Integrin and cadherin signaling in bone: Role and potential therapeutic targets. Trends Endocrinol Metab 25: 567-575, 2014. 